\section{A missing link}

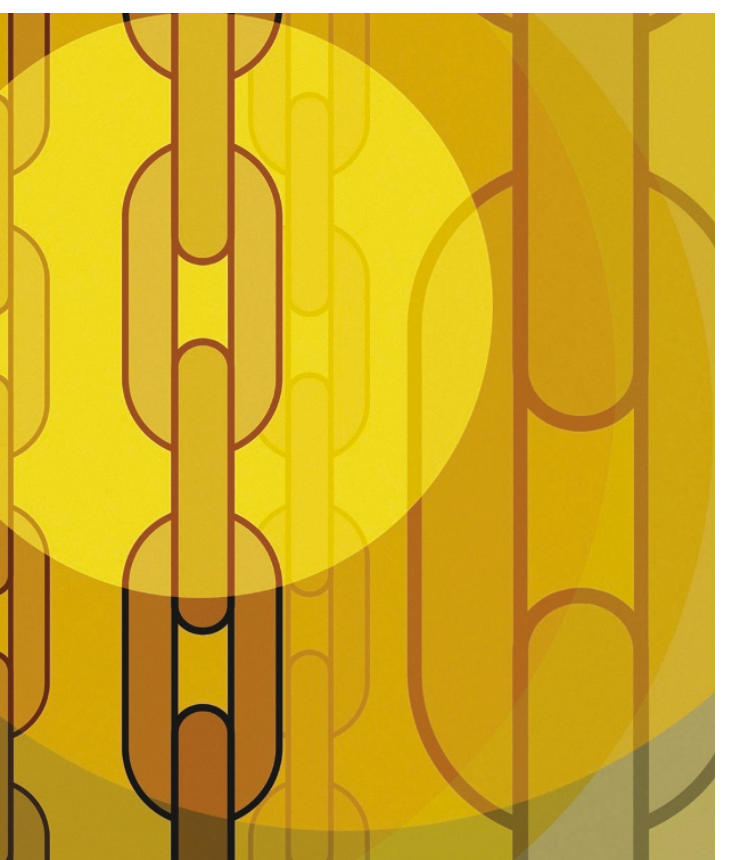

STEM CELLS

\section{Hidden talents}

CD45 is a haematopoietic cell-surface marker that, until recently, wasn't thought to be expressed on any other cell types. So, the observation that muscle-derived $\mathrm{CD} 45^{+}$stem cells can become muscle cells in response to Wnt signalling not only shows that these cells have a biological role in regeneration, but also indicates that targeting Wnt signalling might be effective in treating neuromuscular degenerative disease.

Michael Rudnicki's group, reporting in Cell, observed a tenfold increase in the proportion of cells expressing CD45 and the stem-cell marker Sca-1 (stem cell antigen-1) in regenerating tissues compared to uninjured cells; these CD45 and Sca-1-expressing cells also proliferated extensively. Induction of Myf5, MyoD, desmin and Pax7 was used to show that muscle-derived $\mathrm{CD} 45^{+}-\mathrm{Sca}-1^{+}$cells undergo myogenic specification in response to muscle damage. $\mathrm{CD} 45^{+}-\mathrm{Sca}-1^{+}$cells from uninjured skeletal muscle didn't form myogenic cells, but co-culturing them with primary myoblasts increased the frequency of myogenic differentiation.

Signalling through the Wnt pathway directs cell-fate determination in skeletal muscleWnts initiate myogenesis by inducing the
In higher eukaryotes, $\mathrm{H} 1$ linker histones are involved in the organization of DNA into chromatin, but what other functions they might have is unclear. A study by Steve Jackson, Jessica Downs and colleagues now indicates an unexpected role for the yeast histone H1 homologue (Hhol) in the control of homologous recombination.

The Saccharomyces cerevisiae Hhol protein has a high level of homology to H1 histones, indicating a role in chromatin structure. However, yeast cells that are mutant for HHO1 are viable, grow normally and have no defects in the organization of their DNA. To determine the role of Hho1, Jackson and co-workers studied the effects of exposing cells lacking this protein to a chemical that causes DNA damage. To their surprise, a larger percentage of hho1mutant cells survived than did wild-type cells, which indicates that a lack of Hhol might increase the activity of DNA-repair mechanisms.

To test this, the authors repeated this experiment in cells that were defective for DNA repair by one of two pathways: homologous recombination (HR) or non-homologous end joining (NHEJ). They found that the increased survival in the absence of Hhol was abolished in cells lacking the $R A D 52$ gene, which is required for $\mathrm{HR}$, but increased survival is not abolished in cells defective for NHEJ. This indicated that the function of Hhol in wild-type cells is to suppress HR. In support of this, Jackson and colleagues also found that the overexpression of Hhol caused an increase in sensitivity to DNA damage in wild-type and NHEJ-defective cells, but did not affect the survival of rad52 mutants.

The authors also found effects of Hhol on lifespan and telomere maintenance. Increased levels of recombination cause reduced lifespan in yeast. Consistent with this, the absence of HHO1 had a similar effect, supporting the idea that increased recombination leads to a reduction in longevity.

Jackson and colleagues also provided evidence that Hhol suppresses a telomere maintenance mechanism. In wild-type cells, telomere length is maintained by the enzyme telomerase. However, using an alternative mechanism, cells can prevent telomere shortening through recombination. The authors showed that in cells lacking a crucial component of telomerase, the removal of Hhol resulted in increased survival. This was found to be due to the maintenance of telomeres by a recombinationdependent mechanism. expression of Pax7. As lithium can activate Wnt signalling, Rudnicki's group treated cocultures of $\mathrm{CD}^{2} 5^{+}-\mathrm{Sca}-1^{+}$cells with lithium and saw a 15 -fold increase in myogenic specification; even lithium-treated $\mathrm{CD} 45^{+}-\mathrm{Sca}-1^{+}$cells cultured without myoblasts underwent myogenic differentiation.

Gene-expression studies showed that messenger RNAs for Wnt5a, Wnt5b, Wnt7a and $W n t 7 b$ were induced in regenerating muscle. Further analysis of co-cultures showed that Wnt5a and Wnt $5 \mathrm{~b}$ were expressed in proliferating myoblasts but not differentiated myotubes. Wnt7a, by contrast, was expressed in myotubes but not myoblasts. Wnt5a, Wnt5b and Wnt7a were all expressed in single muscle fibres. Wnt7b mRNA, however, wasn't detected in any sample. So, combined signalling by Wnt $5 a$, Wnt $5 b$ and Wnt7a which are presumably secreted by myofibres and myoblasts in regenerating muscle seems to induce the myogenic commitment of adult muscle-derived stem cells.

If this is true, then $\mathrm{CD} 45^{+}-\mathrm{Sca}-1^{+}$cells would be expected to express the Wnt receptor, Frizzled, which was indeed the case. And, confirming that the Wnt pathway was activated in regenerating muscle, high levels of the Wnt target $\beta$-catenin were found in CD $45^{+}-\mathrm{Sca}-1^{+}$cells. Next, the authors showed that there were increased $\beta$-catenin levels - along with increased MyoD and

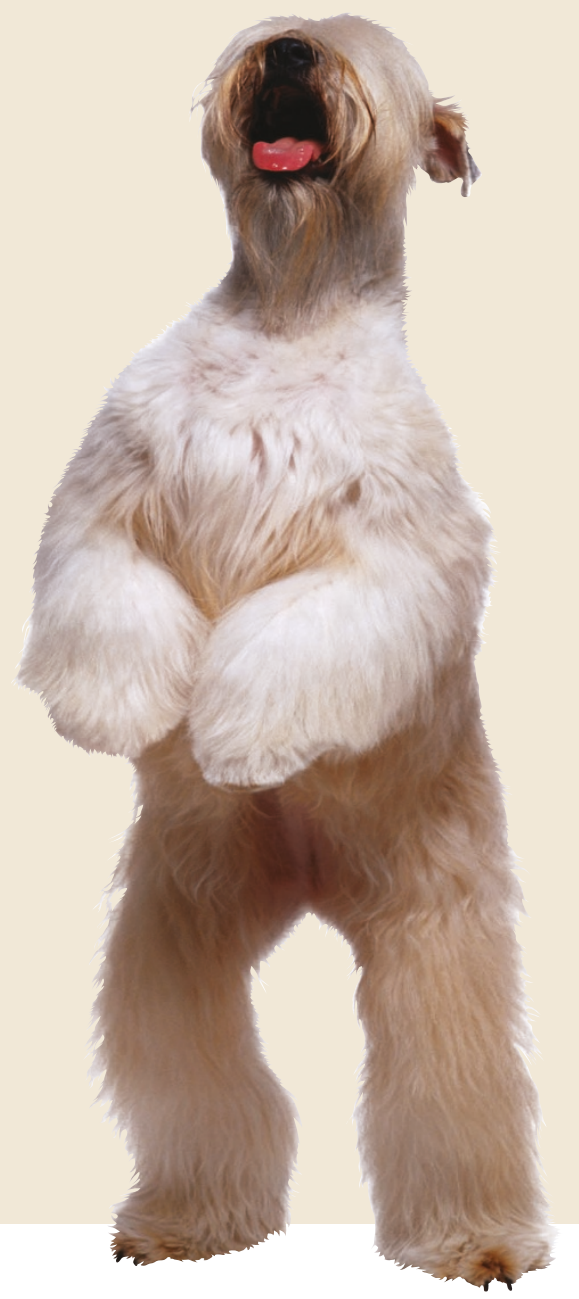


The finding that Hhol functions to inhibit recombination is surprising, as HR is an essential mechanism that is used by cells to repair DNA damage. But inappropriate activation of HR can also be extremely harmful, leading to chromosomal rearrangements that cause genomic instability - and the role of Hhol could be to suppress this.

A second potential function of Hhol might be to prevent cells from carrying out telomerase-independent telomere maintenance. If linker histones from higher eukaryotes are found to have similar functions, this will have important implications for tumorigenesis, which is linked in many cases to genome instability and requires the maintenance of telomere length for immortalization. So, far from being purely structural proteins, $\mathrm{H} 1$ histones might have important roles in cellular processes that are vital for maintaining genome integrity.

Louisa Flintoft, Nature Publishing Group

\section{(2) References and links}

ORIGINAL RESEARCH PAPER Downs, J. A. et al.

Suppression of homologous recombination by the

Saccharomyces cerevisiae linker histone. Mol. Cell 11 1685-1692 (2003)

\section{WEB SITE}

Jessica Down's laboratory:

http://www.bioc.cam.ac.uk/UTOs/Downs.html

Pax7 expression - in CD45 ${ }^{+}-\mathrm{Sca}-1^{+}$cells co-cultured with a stable cell line expressing recombinant Wnt. This confirmed that Wnt signalling activates myogenic determination.

To determine how relevant Wnt signalling is in vivo, Rudnicki and colleagues injected the Wnt antagonists soluble-Frizzled-related proteins (sFRPs) 2 and 3 into regenerating mouse muscle. sFRP2 and sFRP3 inhibited the increase in $\mathrm{CD}^{2} 5^{+}-\mathrm{Sca}-\mathrm{1}^{+}$cells, and the induction of Myf5, that was seen in regenerating cells following injury.

So, the authors propose that $\mathrm{CD} 45^{+}-\mathrm{Sca}-\mathrm{1}^{+}$ cells represent a main source of progenitor cells - thought to be stable and resident in muscles (rather than marrow-derived) that have an important role in muscle regeneration. The molecular mechanisms by which they respond to Wnt signals to induce myogenesis aren't clear, and investigation of the transcriptional targets of Wnts in stem cells will be necessary. But "...the ability of injected sFRP proteins to attenuate the myogenic differentiation of $\mathrm{CD} 45^{+}$stem cells unequivocally underscores the clinical potential for modulating Wnt signalling in muscle tissue".

Katrin Bussell

\section{(4) References and links}

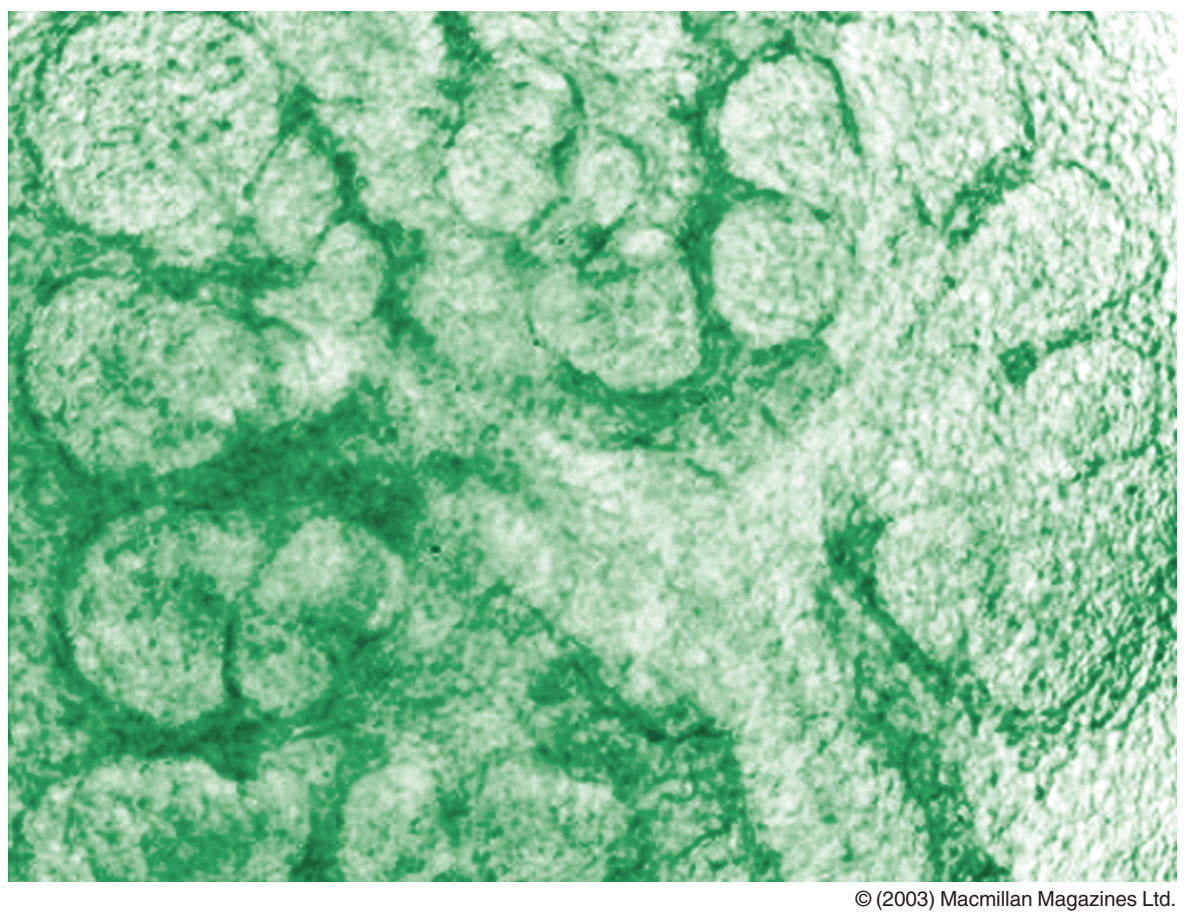

\section{EXTRACELLULAR MATRIX}

\section{A new branch}

One of the wonders of development is the process of branching morphogenesis. Characteristic of many organs such as salivary glands and kidney, complex three-dimensional epithelial branching structures arise from repetitive cleft and bud formation, but the exact mechanisms behind this are not clear. Ken Yamada's group, though, now reports on the essential role of the extracellular matrix (ECM) protein fibronectin in epithelial branching.

ECM components are already known to be required for salivary gland branching, but the authors were interested in identifying locally synthesized regulatory proteins that might influence branching. Because collagen type III accumulates at clefts and fibronectin can regulate collagen III formation, Yamada's group proposed that local fibronectin production might be important in branching morphogenesis, so they searched for differences in fibronectin messenger RNA expression in salivary glands. Quantitative reverse-transcription polymerase chain reaction showed that fibronectin mRNA was expressed at 16-fold higher levels in cleft epithelial cells than in bud epithelium. A closer look showed that cells next to nascent clefts expressed fibronectin mRNA, and high levels of fibronectin fibrils were seen in clefts of branching epithelium. Furthermore, an even closer look showed that the levels of the cell-cell adhesion molecule E-cadherin were lower in the regions right next to the fibronectin fibrils.

So if the accumulation of fibronectin fibrils promoted cleft formation during branching morphogenesis, could inhibiting fibronectin function block branching? Anti-fibronectin antibodies did indeed prevent salivary cleft formation and branching in a dose-dependent manner. Furthermore, antibodies against $\beta_{1}, \alpha_{5}$ or $\alpha_{6}$ integrins inhibited salivary branching. $\alpha_{5} \beta_{1}$ is a key fibronectin receptor, but the fact that combining antibodies against $\alpha_{5}$ and $\alpha_{6}$ more effectively inhibited branching indicated that fibronectin together with laminin $\left(\alpha_{6} \beta_{1}\right.$ is its main receptor in this tissue) might be necessary for branching.

Similar to the results seen when protein function was inhibited, small interfering RNA (siRNA)-mediated knock-down of fibronectin expression in salivary-gland organ culture also markedly decreased cleft formation. Yamada's group then looked at the effects of using exogenous fibronectin to replace the fibronectin. Not only did it successfully restore branching in siRNA-treated salivary glands, but it also stimulated branching in control, non-siRNA-treated cultures in a dose-dependent manner - cleft formation was accelerated and clefts deepened. Similar observations were made in other organs - fibronectin accumulated at sites of indentation, blocking fibronectin function inhibited branching, and exogenous fibronectin promoted branching.

The authors then returned to their previous observation that cadherin levels decreased near presumptive cleft regions, and tested whether fibronectin might induce this decrease. Treating cultured salivary gland epithelial cells with fibrillar fibronectin induced local cell-matrix adhesions, but next to these sites, cadherin localization was suppressed. The predicted resultant loss of cell-cell adhesion would then provide a way for deep clefts to form. And, as well as being responsible for creating the clefts, fibronectin might also maintain them through its ability to regulate collagen, which is thought to be essential for stabilizing clefts.

(2) References and links

Katrin Bussell

\section{ORIGINAL RESEARCH PAPER Sakai, T, Larsen, M. \&}

Yamada, K. Fibronectin requirement in branching morphogenesis. Nature 423, 876-881 (2003) 